\title{
One-Year Intense Nutritional Counseling Results in Histological Improvement in Patients with Nonalcoholic Steatohepatitis: A Pilot Study
}

\author{
Mary Ann Huang, M.D., M.S., Joel K. Greenson, M.D., Cewin Chao, R.D., Lilian Anderson, M.S., R.D., \\ Debra Peterman, R.D., Jon Jacobson, M.D., Dawn Emick, B.S., Anna S. Lok, M.D., \\ and Hari S. Conjeevaram, M.D., M.S. \\ Division of Gastroenterology, Department of Internal Medicine; Department of Pathology; General Clinical \\ Research Center; Department of Radiology, University of Michigan Medical Center, Ann Arbor, Michigan
}

\begin{abstract}
BACKGROUND In individuals with biopsy-proven non-alcoholic steatohepatitis (NASH), short-term weight loss has AND AIM: been shown to improve biochemical abnormalities; however, its effect on liver histology is largely unknown. The aim of the article is to determine if dietary intervention is effective in improving histological features of steatohepatitis in patients with biopsy-proven NASH.
\end{abstract}

METHODS: Twenty-three patients (11M/12F) with BMI $>25 \mathrm{~kg} / \mathrm{m}^{2}$ and biopsy-proven NASH received standardized nutritional counseling aimed at reducing insulin resistance (IR) and weight. Blood tests were checked at baseline and every 1-4 months, and liver biopsy was repeated at month 12. IR was assessed by the homeostasis model assessment (HOMA). Liver biopsies were scored according to modified Brunt criteria for NASH. "Histologic response" was defined as a reduction in total NASH score of $\geq 2$ points with at least one point being in the non-steatosis component.

RESULTS: $\quad$ Sixteen patients $(8 \mathrm{M} / 8 \mathrm{~F})$ completed 12 months of dietary intervention, and 15 underwent repeat liver biopsies. At month 12, mean weight decreased from 98.3 to $95.4 \mathrm{~kg}$. Mean waist circumference, visceral fat, fasting glucose, IR, triglycerides, AST, ALT, and histologic score were all reduced but the difference was not significant. Nine patients had a histologic response, six had stable scores, and none had a worsened score. Compared to patients with unchanged histologic scores, patients with improved scores had significantly greater reduction in weight, waist circumference, AST, ALT, steatosis grade, and total NASH score.

CONCLUSION: Among patients who successfully completed $1 \mathrm{yr}$ of intense dietary intervention, nine of 15 patients with NASH displayed histologic improvement. This pilot study suggests that dietary intervention can be effective in improving histology in patients with biopsy-proven NASH.

(Am J Gastroenterol 2005;100:1072-1081)

\section{INTRODUCTION}

Non-alcoholic steatohepatitis (NASH), also known as fatty liver hepatitis, is increasingly recognized as a common and potentially severe form of liver disease in the United States (1, 2). The term NASH was first coined by Ludwig who described biopsy findings similar to those with alcoholic liver disease (3). NASH is a part of a wide spectrum of clinicopathologic liver disease known as non-alcoholic fatty liver disease or NAFLD. Initially thought to be a benign disease process, NASH may progress to cirrhosis, hepatocellular carcinoma (HCC), and liver failure, sometimes necessitating liver transplantation (4). Recent studies suggest that NASH may be the primary cause of up to $15 \%$ of cryptogenic cirrhosis with an estimated $8-26 \%$ progressing to cirrhosis $(5,6)$.

Although the exact mechanism for the development of NAFLD is not known, insulin resistance (IR) has been im- plicated as the key factor even among patients not known to be obese or diabetic. Recent studies showed that patients with NASH have a higher prevalence of IR compared to controls and patients with chronic hepatitis $\mathrm{C}$. Though IR was identified in patients with normal BMI and anthropometric measurements, the majority of these patients had abdominal adiposity suggesting that increased visceral fat known to cause IR may also be an important factor in the development of hepatic steatosis $(7,8)$.

The natural history and progression of NASH is not well characterized. However, available data suggest that while patients with steatosis alone tend to have a relatively benign course, patients with NASH are at risk for progression to fibrosis and cirrhosis. At present, there is no established means for treating or preventing NASH. In overweight or obese individuals, weight loss is usually recommended as the first line management, but this is rarely achieved or maintained 
in clinical practice. Short-term weight loss (diet-induced or exercise-induced) has been shown to improve IR, decrease visceral fat, and hepatic steatosis; however, its ultimate effect on liver histology and the natural course of NASH is unknown (9). Most studies to date have been short-term and included small number of patients and in some series weight loss was achieved with gastric bypass surgery in morbidly obese patients (10-13). More recently, pilot studies using the insulin sensitizing drugs belonging to the class of thiazolidinediones have shown histologic improvement but only small case series have been reported $(14,15)$. The beneficial effects were not sustained and weight gain was observed in up to $72 \%$ of patients.

The aim of this study was to assess the effect of a 1-yr intense dietary counseling targeted toward reducing IR on liver histology in overweight and obese patients with biopsyproven NASH.

\section{METHODS}

A prospective single arm pilot study of dietary intervention in patients with biopsy-proven NASH seen in the liver clinics at the University of Michigan was performed. Adult patients with a liver biopsy demonstrating NASH within $1 \mathrm{yr}$ of enrollment, a BMI greater than $25 \mathrm{~kg} / \mathrm{m}^{2}$, no other etiologies of chronic liver disease, alcohol use $<20 \mathrm{~g}$ a day, and an elevation in serum aspartate aminotransferase (AST) or alanine aminotransferase (ALT) above the upper limit of normal on at least one occasion as defined by our hospital laboratory manual were included. Patients with other causes of chronic liver disease including viral hepatitis, autoimmune hepatitis, cholestatic liver disease, alpha-1 antitrypsin deficiency, hemochromatosis, Wilson's disease, and alcoholic liver disease were excluded based on history, laboratory results, and histologic features. The patients on medications known to cause hepatic steatosis, had undergone weightreduction surgery within the past year, or used weight loss medications within 3 months of evaluation, were also excluded from participation in this study.

The study was approved by the Institutional Review Board (IRB) at the University of Michigan on October 1, 2001. Eligible subjects were screened by a dietitian to assess their willingness to comply with the 12-month intervention program after written informed consent was obtained. The protocol included visits with the dietitians detailed below; clinical assessments every 1-2 months; liver panel every $1-2$ months; lipid profile, fasting insulin, and glucose every 4 months; pre- and postintervention single slice $\mathrm{CT}$ scans to measure visceral adiposity; and a follow-up liver biopsy at the end of the intervention program. Waist and hip measurements were obtained by a Registered Dietitian at baseline, and then every 2 months. Weight and blood pressure were obtained at every scheduled visit.

\section{Dietary Intervention}

All participants received standardized nutritional counseling directed at decreasing IR and promoting gradual weight loss
(1-2 pounds/wk) over the duration of the study. The participants were seen weekly by a Registered Dietitian for the first 8 wk, biweekly from months $3-6$, and then monthly from months 6-12. In addition, participants were invited to attend a total of four group sessions. At the baseline visit, nutritional assessment included food recalls to assess actual dietary intake. Food frequency questionnaires (FFQ) were obtained to assess usual dietary intake. The Paffenbarger Activity Questionnaire (PAQ) was used to assess the level of physical activity. Body impedance analysis (BIA) was obtained to evaluate body composition along with baseline weight and baseline laboratory studies. During the follow-up visits, each subject received individualized nutrition counseling in order to achieve dietary goals. The dietary adjustments included increased intake of fiber and decreased calories, and advice on increasing physical activity. Each participant was encouraged to increase his/her activity with the goal of achieving a heart rate of $70 \%$ of the calculated target heart rate. The recommendations were individualized per patient, and the PAQ was repeated at the end of the study to assess whether the participants changed their level of activity.

The diet selected to reduce IR was as follows:

- $40-45 \%$ of daily calories from carbohydrates with an emphasis on complex carbohydrates with fiber,

- $35-40 \%$ fat with emphasis on mono- and polyunsaturated fats, and

- $15-20 \%$ protein.

This macronutrient breakdown is similar to that of the Syndrome X diet, designed by Dr. Gerald Reaven (16). Dietary instructions also took into consideration any concurrent diagnoses, such as the presence of diabetes, hypertension, and coronary artery disease.

The study required the patients to maintain records about their food intake and activity. The program mandated that the patients attend all sessions with the study dietitian and be compliant with all clinical visits. For each patient, weekly food records and self-reported intakes were serially assessed and analyzed for their nutritional composition and compared to the goals for the individual patient.

\section{Single Slice CT Scan}

Quantitative CT (Model High Speed CSI, GE Medical Systems, Milwaukee, WI) was performed for the measurement of abdominal visceral and non-visceral fat. Individuals with visceral adipose tissue cross-sectional area of $>100 \mathrm{~cm}^{2}$ were classified as visceral obesity (17). A non-contrast $1-\mathrm{cm}$ axial section was obtained through the mid aspect of the fourth lumbar vertebral body parallel to both endplates. Regions of interest were proscribed (J.J.) using graphical image analysis software (GE Advance Windows Workstation, Version 4.1, Milwaukee, WI) and cross-sectional fat area for each abdominal compartment was defined as the summated area of pixels that have attenuation coefficients between -50 and -250 Hounsfield units. The internal most aspect of the abdominal and oblique muscle walls and psoas muscles and the 
Table 1. Modified Brunt Criteria of Staging and Grading NAFLD

\begin{tabular}{|c|c|}
\hline Steatosis & Score $(\operatorname{Max}=4)$ \\
\hline Macrovesicular steatosis & $\begin{array}{l}0 \text { - None } \\
\text { 1-Minimal }(<10 \%) \\
\text { 2-Mild }(10-33 \%) \\
\text { 3-Moderate }(33-66 \%) \\
\text { 4-Severe }(>66 \%)\end{array}$ \\
\hline $\begin{array}{l}\text { Hepatitis (ballooning and } \\
\text { inflammation) }\end{array}$ & Score $(\max =9)$ \\
\hline \multirow{2}{*}{$\begin{array}{l}\text { Hepatocellular ballooning and } \\
\text { disarray }\end{array}$} & 0 - None \\
\hline & $\begin{array}{l}\text { 1-Occasional ballooning } \\
\text { of zone } 3 \text { hepatocytes } \\
2 \text { - Obvious ballooning of } \\
\text { hepatocytes } \\
\text { 3-Obvious ballooning } \\
\text { and disarray of } \\
\text { hepatocytes }\end{array}$ \\
\hline \multirow[t]{2}{*}{$\begin{array}{l}\text { Intra-acinar (lobular) } \\
\text { inflammation (number of } \\
\text { inflammatory foci with a } 20 \times \\
\text { ocular) }\end{array}$} & 0 - None \\
\hline & $\begin{array}{l}1-1 \text { to } 2 / 20 \times \\
2-\text { up to } 4 / 20 \times \\
3->4 / 20 \times\end{array}$ \\
\hline Portal tract inflammation & $\begin{array}{l}0 \text { - None } \\
\text { 1-Mild } \\
\text { 2-Moderate } \\
\text { 3-Severe }\end{array}$ \\
\hline Staging (fibrosis) & Score $(\max =4)$ \\
\hline Fibrosis score & $\begin{array}{l}0 \text { - None } \\
\text { 1-Zone } 3 \text { perisinu- } \\
\text { soidal/pericellular } \\
\text { fibrosis; focally or } \\
\text { extensively present } \\
\text { 2-Zone } 3 \text { perisinu- } \\
\text { soidal/pericellular } \\
\text { fibrosis with focal or } \\
\text { extensive periportal } \\
\text { fibrosis } \\
\text { 3-Zone } 3 \text { perisinu- } \\
\text { soidal/pericellular } \\
\text { fibrosis, and portal } \\
\text { fibrosis with focal or } \\
\text { extensive bridging } \\
\text { fibrosis } \\
\text { 4-Cirrhosis }\end{array}$ \\
\hline
\end{tabular}

Maximum total score $=17$

anterior aspect of the vertebral body were used as borders for visceral fat area. Abdominal subcutaneous fat mass was calculated as the difference between total abdominal fat and the intraabdominal compartment fat within the deep fascia (18).

\section{Liver Biopsy}

The diagnosis of NASH was based on findings of hepatocellular steatosis and ballooning, mixed acute and chronic lobular inflammation, and zone 3 perisinusoidal fibrosis. All the biopsies were read by one hepatopathologist (J.K.G.) who utilized a modified Brunt scoring system for NASH (Table 1). The modifications included expanding the steatosis scoring from $0-3$ to $0-4$ and a numerical score for hepatocellular ballooning and disarray (Table 1). The maximum NASH histological score was 17 .

\section{Insulin Resistance}

The homeostasis model assessment (HOMA) to calculate IR $(\mathrm{HOMA}=($ insulin $(\mathrm{uU} / \mathrm{ml}) *$ glucose $(\mathrm{mmol} / \mathrm{L})) / 22.5)$ was utilized. A HOMA value of 1.64 or greater was defined as IR (7).

\section{End Point of Study}

The primary end point in our study was histologic improvement defined as $\geq 2$-point reduction in the total NASH score with at least one point being in the non-steatosis component.

\section{Statistical Analysis}

Statistical analysis was performed using the SAS software (Version 8e, Cary, NC). All patient data were stored in an Excel database on a password-protected computer.

When calculating sample size for this pilot study, there was no previous histologic data in the literature to follow. So our hypothesis was that dietary intervention would produce a histologic improvement of $\geq 2$ points of the total NASH score in $20 \%$ of the subjects, which would produce a clinically meaningful result. With a two-sided $p$-value of 0.01 and power of 0.80 , a minimum of 10 patients was required to complete $1 \mathrm{yr}$ of therapy. We anticipated that about $70 \%$ of the patients would complete the intense dietary program and $70 \%$ of patients who completed the program to consent to a repeat biopsy; therefore, we estimated 20 patients would need to be initially enrolled.

\section{RESULTS}

From October 2001 to November 2002, a total of 37 patients were initially screened for inclusion in the study. After excluding 17 patients for normal BMI $(\mathrm{n}=3)$, normal AST/ALT $(n=4)$, or refusal for inclusion in the study $(n=10), 20$ patients were initially enrolled. Three patients dropped out within the first 2 months; these three patients were replaced. Thus, a total of 23 patients were enrolled. Four additional patients dropped out later in the study. In total, seven patients dropped out. The reasons included the following: three had difficulty adhering to the frequent study visits due to family and personal problems, two were lost to follow-up, one moved out of state, and one became pregnant (an exclusion criteria in the study). Sixteen patients successfully completed the 1 yr intervention and 15 agreed to a follow-up liver biopsy.

Baseline characteristics of the patients are shown in Table 2. All 23 patients who were enrolled had evidence of IR at entry. There was no difference at baseline between the 16 patients who did and the other 7 patients who did not complete the $1 \mathrm{yr}$ dietary intervention. The comparison of baseline and 
Table 2. Baseline Characteristics of all Enrolled Patients $(n=23)$

\begin{tabular}{lccc}
\hline Parameters & All Patients & 16 Patients Who Completed Study & Seven Patients Who Dropped Out \\
\hline Age (yr) & $47.8( \pm 12)$ & $49.8( \pm 12)$ & $43.3( \pm 12)$ \\
Gender & $11 \mathrm{M} / 12 \mathrm{~F}$ & $8 \mathrm{M} / 8 \mathrm{~F}$ & $3 \mathrm{M} / 4 \mathrm{~F}$ \\
Ethnicity & $20 \mathrm{NHW}, 3$ Hispanic & $14 \mathrm{NHW}, 2 \mathrm{Hispanic}$ & $6 \mathrm{NHW}, 1 \mathrm{Hispanic}$ \\
Weight (kg) & $97.4( \pm 18)$ & $98.3( \pm 20)$ & $95.2( \pm 16)$ \\
BMI (kg/m ${ }^{2}$ & $33.8( \pm 6)$ & $34.0( \pm 7)$ & $33.3( \pm 4)$ \\
No. (\%) with diabetes mellitus & $4(17 \%)$ & $2(12.5 \%)$ & $2(29 \%)$ \\
Waist circumference (cm) & $105.4( \pm 13)$ & $106.6( \pm 13)$ & $102.4( \pm 11)$ \\
Visceral fat (cc) & $197.0( \pm 55)$ & $31.3( \pm 10)$ & $210.5( \pm 54)$ \\
Body fat by BIA (\%) & $35.3( \pm 13)$ & $6.1( \pm 6)$ & $42.2( \pm 15)$ \\
HOMA score & $6.6( \pm 5)$ & $59.9( \pm 31)$ & $7.8( \pm 3)$ \\
AST (IU/L) & $60.7( \pm 32)$ & $81.8( \pm 38)$ & $62.4( \pm 37)$ \\
ALT (IU/L) & $89.3( \pm 42)$ & $43.0( \pm 14)$ & $106.3( \pm 49)$ \\
HDL (mg/dL) & $41.6( \pm 13)$ & $212.1( \pm 131)$ & $38.3( \pm 12)$ \\
Triglycerides (mg/dL) & $200.3( \pm 112)$ & $9.2( \pm 3)$ & $173.4( \pm 44)$ \\
Baseline total NASH score & $9.1( \pm 3)$ & & $9( \pm 2)$ \\
\hline
\end{tabular}

BMI, body mass index; HOMA, homeostasis model assessment for insulin resistance.

AST, aspartate aminotransferase; ALT, alanine aminotransferase; HDL, high density lipid fraction of cholesterol.

Upper limit of normal for AST $=35 \mathrm{IU} / \mathrm{L}$ and for ALT $=45 \mathrm{IU} / \mathrm{L}$.

${ }^{*}$ There was no significant difference between the 16 patients who completed 12 months of intervention and the 7 patients who dropped out before the end the study for all baseline parameters.

month 12 parameters among the 15 patients who had a followup liver biopsy showed a significant improvement in IR based on HOMA index $(6.5+5$ vs $3.4+2, p=0.02)$. In addition, mean body weight, BMI, waist circumference, total body fat by BIA, visceral fat volume on single slice CT, serum AST, ALT, and triglyceride levels also decreased from baseline to month 12 in these 15 patients who completed the study, but these differences did not reach statistical significance (Table 3, Figs. 1-3).

Nine of 15 (60\%) patients who successfully completed the 1-yr dietary intervention and had a follow-up liver biopsy showed a histologic response, six had stable NASH scores,

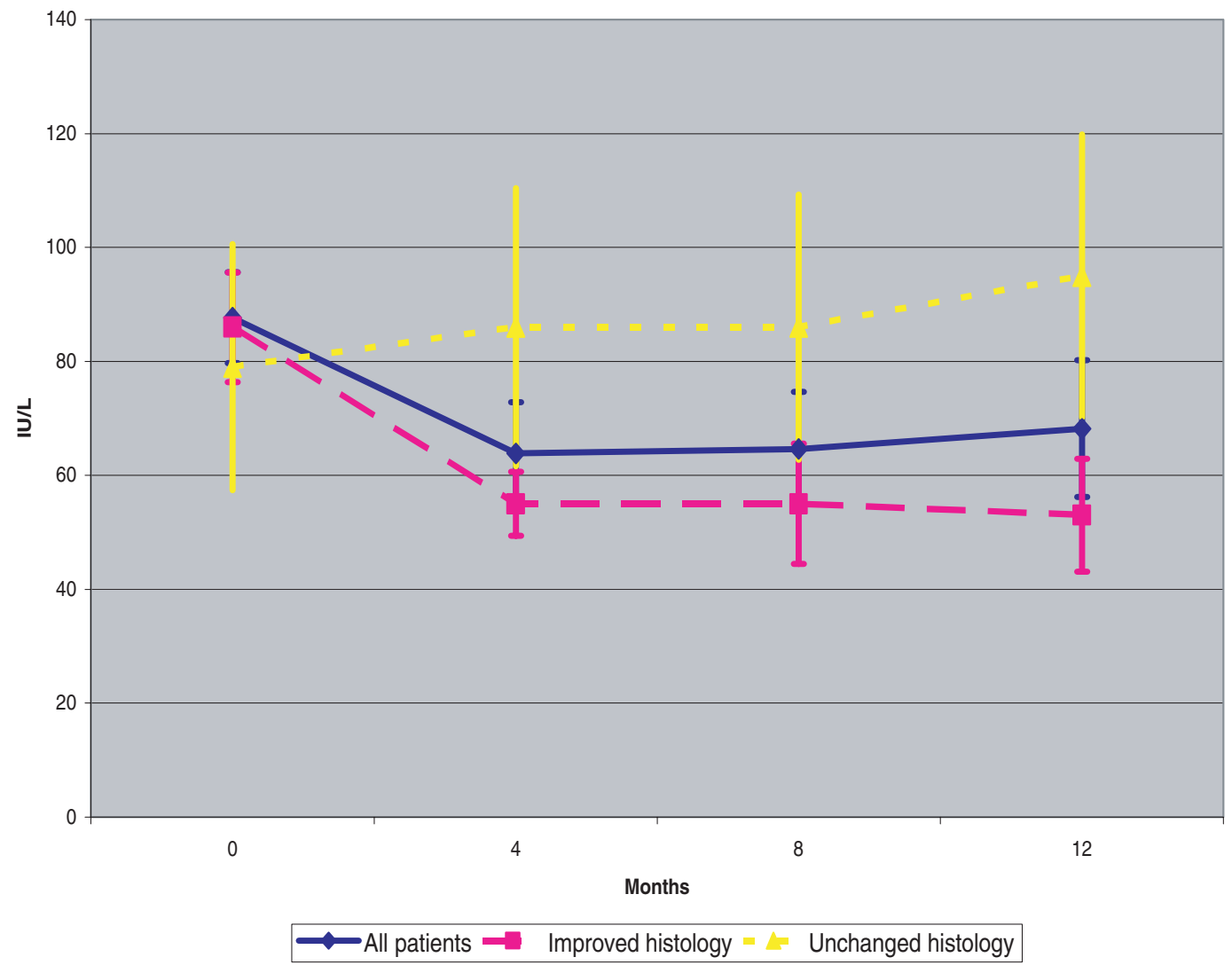

Figure 1. Mean serum ALT over time ( \pm SEM). 


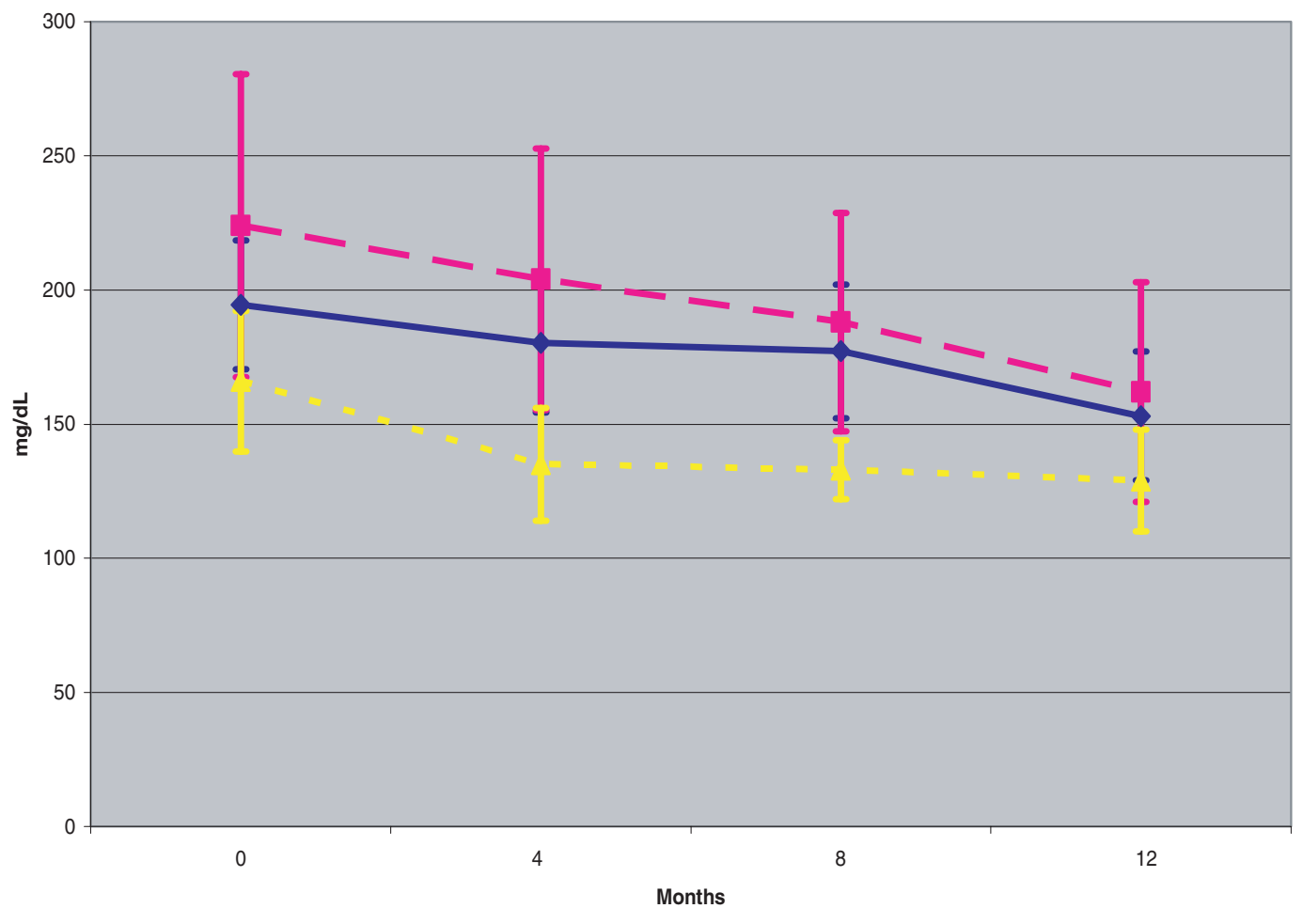

$\longrightarrow$ All patients $\longrightarrow$ Improved histology $=$ Unchanged histology

Figure 2. Mean serum triglyceride levels of all subjects over time ( \pm SEM).

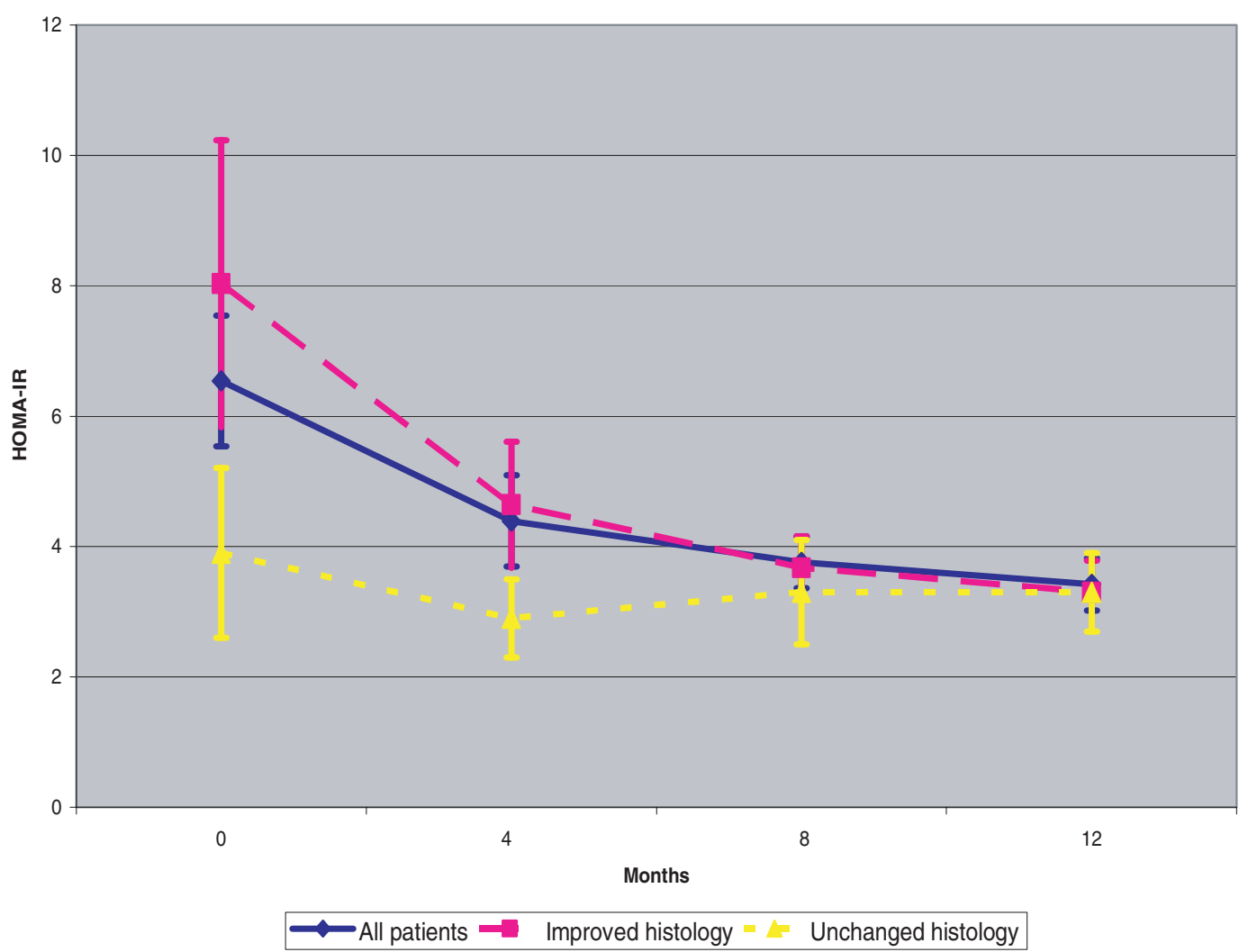

Figure 3. Mean HOMA-IR score of all subjects over time ( \pm SEM). 
Table 3. Comparison of Baseline to End-of-Study Parameters in the 15 Patients Who Completed the Study and Underwent Repeat Liver Biopsies

\begin{tabular}{lccc}
\hline Parameter & Baseline & Month 12 & $p$-Value \\
\hline Weight $(\mathrm{kg})$ & $99.3( \pm 20)$ & $96( \pm 21)$ & 0.7 \\
BMI $\left(\mathrm{kg} / \mathrm{m}^{2}\right)$ & $34.4( \pm 7)$ & $33.3( \pm 7)$ & 0.7 \\
Waist circumference $(\mathrm{cm})$ & $107( \pm 14)$ & $103( \pm 14)$ & 0.6 \\
Visceral fat $(\mathrm{cc})$ & $191( \pm 57)$ & $165( \pm 45)$ & 0.18 \\
Body fat by BIA $(\%)$ & $35.3( \pm 13)$ & $36.8( \pm 13)$ & 0.7 \\
HOMA-IR & $6.2( \pm 6)$ & $3.3( \pm 1)$ & 0.06 \\
AST (IU/L) & $60( \pm 32)$ & $56( \pm 37)$ & 0.8 \\
ALT (IU/L) & $83( \pm 39)$ & $70( \pm 48)$ & 0.4 \\
HDL (mg/dL) & $44( \pm 14)$ & $48( \pm 17)$ & 0.5 \\
TG (mg/dL) & $210( \pm 136)$ & $149( \pm 98)$ & 0.17 \\
Steatosis score $(0-4)$ & $2.7( \pm 0.9)$ & $2.2( \pm 1.1)$ & 0.18 \\
Hepatitis score $(0-9)$ & $4.4( \pm 2)$ & $3( \pm 2)$ & 0.06 \\
Fibrosis score $(0-4)$ & $2.2( \pm 0.9)$ & $2.3( \pm 0.6)$ & 0.7 \\
Total NASH score $(0-17)$ & $9.3( \pm 3)$ & $7.5( \pm 3)$ & 0.11 \\
\hline
\end{tabular}

and none had a worsening of the NASH score. Figure 4 illustrates the baseline and repeat liver biopsies of a patient who had a histologic response. The changes in metabolic, laboratory, and histologic characteristics of the patients with improved versus those with stable NASH scores are shown in Table 4. The breakdown of the changes in the histologic characteristics (steatosis, hepatitis, fibrosis, and total score) of the 15 patients with follow-up biopsies are shown in Table 5.

Compared to those with stable NASH scores $(\mathrm{n}=6)$, patients with histologic response $(n=9)$ had significantly greater decrease in body weight, BMI, waist circumference, AST, and ALT values (Table 4, Fig. 1). The subjects with histologic response lost an average of $7 \%$ of their body weight (range $=+0.07 \%$ to $-14 \%$ ) compared to nonresponders who gained an average of $2 \%$ body weight (range $=+11 \%$ to $-4 \%)$. Among the patients with histologic response, most of the weight loss occurred in the first 4 months of the study with a more gradual continued weight loss until the end of study. In addition, there was a trend toward a greater reduction in serum TGL levels $(-75.89 v s-37.17)$, IR (HOMA index $-4.6 v s-0.42)$, and visceral fat on CT $(-36.18 \mathrm{cc} v s$ $-12.23 \mathrm{cc}$ ) among the patients with histologic response; however, this did not reach statistical significance (Table 4, Figs. 2-3). All patients had evidence of IR based on HOMA index at baseline although patients with a "histological response" had higher baseline HOMA values compared to those without a response. All nine patients with a histologic response had improvement in IR defined as a decrease in the end of study HOMA score from the initial score compared to only two of six patients with no histologic response $(p<0.05)$. Seven patients with improved histology also had a decrease in the volume of visceral fat compared to three of six patients with no histological response $(p=0.15)$. Figure 5 shows the reduction in visceral fat in one patient who had a histologic response.

Overall, there was a significant reduction in the steatosis (mean change $-1.0 v s+0.17, p<0.01$ ) and total NASH
Table 4. Comparison of Subjects with Histological Responders (Improved NASH Scores) versus Nonresponders (Stable NASH Scores)

\begin{tabular}{|c|c|c|c|}
\hline Parameters & $\begin{array}{c}\text { Patients with } \\
\text { Improved NASH } \\
\text { Score } \\
(\mathrm{n}=9)\end{array}$ & $\begin{array}{l}\text { Patients with } \\
\text { Stable } \\
\text { NASH Score } \\
(n=6)\end{array}$ & $p$-Value \\
\hline Age & $47.1 \pm 11$ & $50.4 \pm 12$ & NS \\
\hline Number of males & $5(56 \%)$ & $3(50 \%)$ & NS \\
\hline $\begin{array}{l}\text { Mean } \Delta \text { in weight } \\
\quad(\mathrm{kg})\end{array}$ & -6.63 & +1.8 & 0.002 \\
\hline $\begin{array}{l}\text { Mean } \Delta \text { in BMI } \\
\left(\mathrm{kg} / \mathrm{m}^{2}\right)\end{array}$ & -2.25 & +0.58 & 0.001 \\
\hline $\begin{array}{l}\text { Mean } \Delta \text { in waist } \\
\text { circumference } \\
(\mathrm{cm})\end{array}$ & -6.94 & -0.52 & 0.01 \\
\hline $\begin{array}{l}\text { Mean } \Delta \text { in visceral } \\
\text { fat }(\mathrm{cc})\end{array}$ & -36.18 & -12.23 & 0.28 \\
\hline $\begin{array}{l}\text { Mean } \Delta \text { in body fat } \\
\text { by BIA }(\%)\end{array}$ & +0.63 & +1.1 & 0.82 \\
\hline $\begin{array}{l}\text { Mean } \Delta \text { in HOMA } \\
\text { score }\end{array}$ & -4.60 & -0.42 & 0.15 \\
\hline $\begin{array}{l}\text { Mean } \Delta \text { in AST } \\
(\mathrm{IU} / \mathrm{L})\end{array}$ & -15.44 & +12.50 & 0.05 \\
\hline $\begin{array}{l}\text { Mean } \Delta \text { in ALT } \\
(\mathrm{IU} / \mathrm{L})\end{array}$ & -33.11 & +15.67 & 0.02 \\
\hline $\begin{array}{l}\text { Mean } \Delta \text { in HDL } \\
\text { level }\end{array}$ & +5.11 & +3 & 0.56 \\
\hline Mean $\Delta$ in TG level & -75.89 & -37.17 & 0.48 \\
\hline $\begin{array}{l}\text { Mean } \Delta \text { in steatosis } \\
\text { score }(0-4)\end{array}$ & -1 & +0.17 & 0.003 \\
\hline $\begin{array}{l}\text { Mean } \Delta \text { in hepatitis } \\
\text { score }(0-9)\end{array}$ & -1.78 & -0.83 & 0.077 \\
\hline $\begin{array}{l}\text { Mean } \Delta \text { in fibrosis } \\
\text { score }(0-4)\end{array}$ & -0.22 & +0.5 & 0.086 \\
\hline $\begin{array}{l}\text { Mean } \Delta \text { in total } \\
\text { NASH score } \\
(0-17)\end{array}$ & -3 & -1.67 & $<0.0001$ \\
\hline
\end{tabular}

score (mean change $-3.0 v s-1.67, p<0.0001$ ) among the responder group compared to those with no response (Table 4). There was also a trend toward a greater reduction in both the hepatitis score $(-1.78 v s-0.83, p=0.07)$ and fibrosis score $(-0.22 v s+0.5, p=0.08)$ between the two groups; however, once again this difference was not significant.

Analysis of the paired biopsies using non-parametric means (Wilcoxon signed rank test) revealed similar results to previously summarized analysis of the mean values using $t$-test. Steatosis score $(p=0.05)$ and total NASH score $(p=$ 0.002 ) were significantly different. In addition, hepatitis score was also significant $(p=0.0005)$ while fibrosis score did not achieve statistical significance.

All the subjects who completed the study showed good compliance with the dietary recommendations, as calculated from food diaries maintained by the subjects. The FFQs showed that the average intake by the subjects was similar to the average American diet as shown by National Health and Nutrition Examination Survey (NHANES) IV. Of the 16 subjects who completed the study, the mean decrease in daily 
Table 5. Changes in Each Component of the NASH Score for the 15 Patients with Paired Biopsies

\begin{tabular}{|c|c|c|c|c|c|c|c|c|}
\hline \multirow[b]{2}{*}{ Subject No. } & \multicolumn{2}{|c|}{ Steatosis Score } & \multicolumn{2}{|c|}{ Hepatitis Score } & \multicolumn{2}{|c|}{ Fibrosis Score } & \multicolumn{2}{|c|}{ NASH Score } \\
\hline & Baseline & Month 12 & Baseline & Month 12 & Baseline & Month 12 & Baseline & Month 12 \\
\hline \multicolumn{9}{|c|}{ Improved histology } \\
\hline 2 & 3 & 1 & 3 & 2 & 1 & 2 & 7 & 5 \\
\hline 3 & 3 & 1 & 3 & 1 & 2 & 2 & 8 & 4 \\
\hline 6 & 3 & 2 & 4 & 2 & 2 & 2 & 9 & 6 \\
\hline 7 & 3 & 2 & 4 & 3 & 3 & 2 & 10 & 7 \\
\hline 8 & 3 & 2 & 5 & 3 & 2 & 2 & 10 & 7 \\
\hline 9 & 3 & 3 & 7 & 5 & 3 & 3 & 13 & 11 \\
\hline 11 & 1 & 1 & 4 & 1 & 3 & 3 & 8 & 5 \\
\hline 13 & 2 & 1 & 2 & 1 & 2 & 1 & 6 & 3 \\
\hline 14 & 2 & 1 & 3 & 1 & 3 & 2 & 8 & 4 \\
\hline \multicolumn{9}{|c|}{ Unchanged histology } \\
\hline 1 & 4 & 4 & 3 & 3 & 2 & 2 & 9 & 9 \\
\hline 4 & 4 & 4 & 7 & 6 & 3 & 3 & 14 & 13 \\
\hline 5 & 2 & 3 & 4 & 4 & 2 & 2 & 8 & 9 \\
\hline 10 & 2 & 2 & 8 & 7 & 3 & 3 & 13 & 12 \\
\hline 12 & 4 & 4 & 7 & 5 & 2 & 3 & 13 & 12 \\
\hline 15 & 2 & 2 & 2 & 1 & 0 & 2 & 4 & 5 \\
\hline
\end{tabular}

caloric intake was $195 \mathrm{kcals}$, mean fat intake was decreased by $3 \%$ of daily calories, carbohydrate intake was maintained, mean fiber intake increased by $1 \mathrm{~g} /$ day, and mean protein intake was increased by $1 \%$ of daily calories. The patients with improved histology tended to have a greater reduction in carbohydrate $(\mathrm{CHO})$ intake with $\mathrm{CHO}$ :protein ratio decreasing from 3.17 to 3.02 and $\mathrm{CHO}$ :fat ratio from 1.7 to 1.64 while patients with unchanged histology increased their $\mathrm{CHO}$ :protein ratio from 2.7 to 3.6 and $\mathrm{CHO}$ :fat ratio from 1.24 to 1.4. Their differences were not statistically significant.

Only 9 of 15 patients who both completed the study and had follow-up histology submitted their activity questionnaires at the end of the 48-wk period ( 6 of 9 responders, 3 of 6 nonresponders). A ratio of post- to pre-intervention activity level was calculated for each subject. Any subject with suboptimal data or a missing questionnaire was excluded. Logistic regression was performed, which showed that patients with histologic response significantly increased the amount of physical activity at the end of the study compared to baseline $(p=0.035)$.

\section{DISCUSSION}

In this study, we showed that a 1-yr dietary program aimed at reducing IR and promoting weight loss resulted in improved histology in $60 \%$ of patients with biopsy-proven NASH who successfully completed the program. Based on an intentionto-treat analysis, the histologic response was seen in $45 \%$ $(9 / 20)$. The dietary program we used was intense as it required the patients to attend frequent clinic visits, maintain records of their food intake and physical activity, have regular blood tests, and undergo a follow-up liver biopsy. We estimate that the cost of an intervention similar to the one utilized in this study to be $\$ 2000$ per patient per year. Much of this expense would be out of pocket for the patient as most insurance companies would not currently pay for such intense dietary counseling. However, the results do show that if patients are motivated, lose weight, and have improvement of insulin sensitivity, it is possible to see histologic improvement.

Although the goal of the dietary intervention was to achieve changes in macronutrient composition targeted to reduce IR, there was not a significant difference in the pre- and postintervention dietary composition among the histologic responders versus nonresponders. The histologic responder group, however, had an average of $7 \%$ weight loss compared to an average of $2 \%$ weight gain in the nonresponders, which points toward weight loss as the major contributor toward histologic improvement $(p<0.01)$. The patients with histologic improvement also had a greater degree of weight loss than patients with stable NASH scores. Weight loss of $5 \%$ total body weight has been shown to improve liver enzymes (19). In this study, those with histologic improvement lost an average of $7 \%$ total body weight with seven of nine patients losing at least $5 \%$. Only one of six patients with unchanged histology lost weight ( $p=0.04$, Fisher's exact test). In addition, physical activity as measured by PAQ was also greater among the histologic responders. However, this was available only in a small number of patients who completed the study. Physical activity can help maintain the weight loss and also improve IR. Whether this effect contributed to the histological response seen in the follow-up liver biopsies is unclear given the limited available data of these patients.

As expected, in this short-term study the greatest improvement in liver histology was the reduction in steatosis. Decrease in hepatitis score by at least 1 point was also observed in three patients and by 2 or more points in six patients indicating that alterations in diet with or without weight 

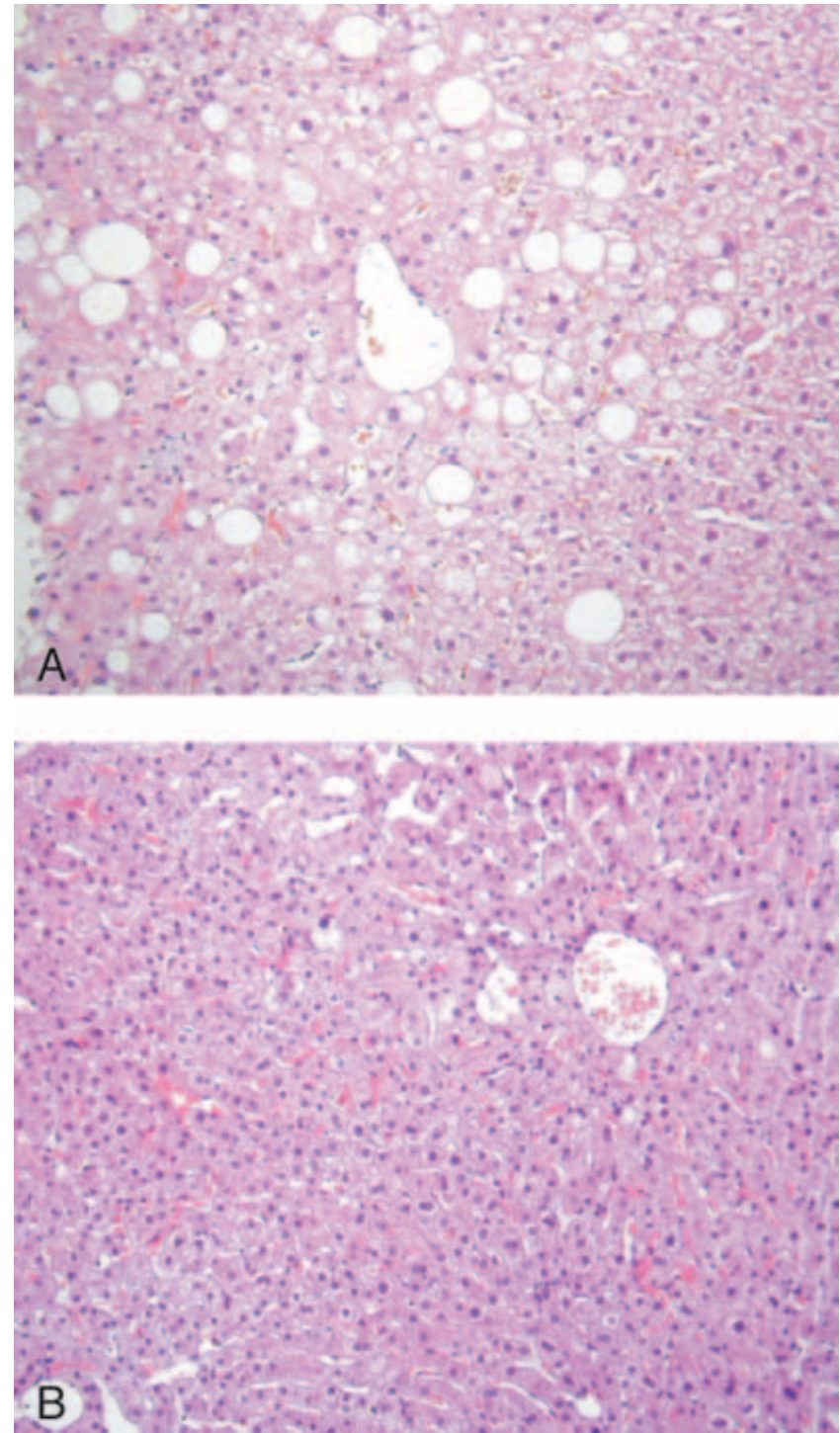

Figure 4. Liver biopsies of patient 13 with histologic response. (A) Baseline liver biopsy: Medium power view of pericentral macrovesicular steatosis from patient in Figure 5. Total NASH score of 6 . $(B)$ Month 12 liver biopsy: Medium power view of pericentral region showing markedly reduced steatosis compared to $6 \mathrm{a}$. Total NASH score of 3 .

loss was effective in decreasing hepatocyte ballooning and inflammation. Only three patients had decrease in fibrosis score, which is likely related to the short duration of the study.

Very limited data are available on the efficacy of nonpharmacologic and pharmacologic interventions on liver histology, particularly inflammation and fibrosis in patients with NASH. Three pilot studies using thiazolidinediones as treatment in patients with biopsy-proven NASH have been published. The first study, using troglitazone, treated 10 women with biopsy-proven NASH for up to 6 months. Liver biopsies were repeated only in seven patients who had normalization of ALT, four showed a 1-point improvement in necroin-
A

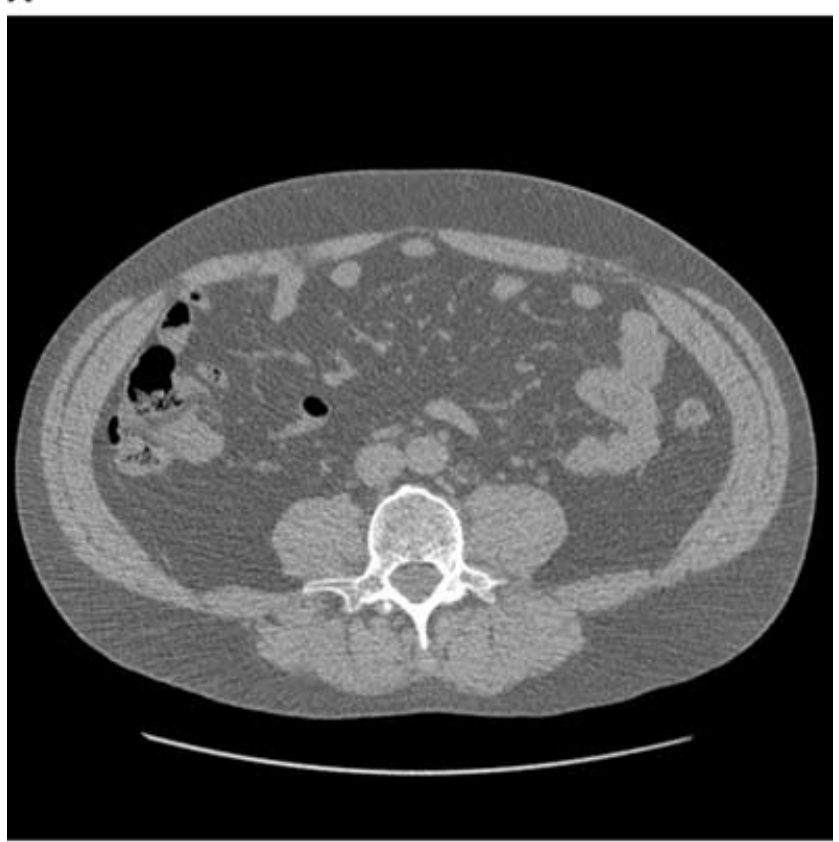

B

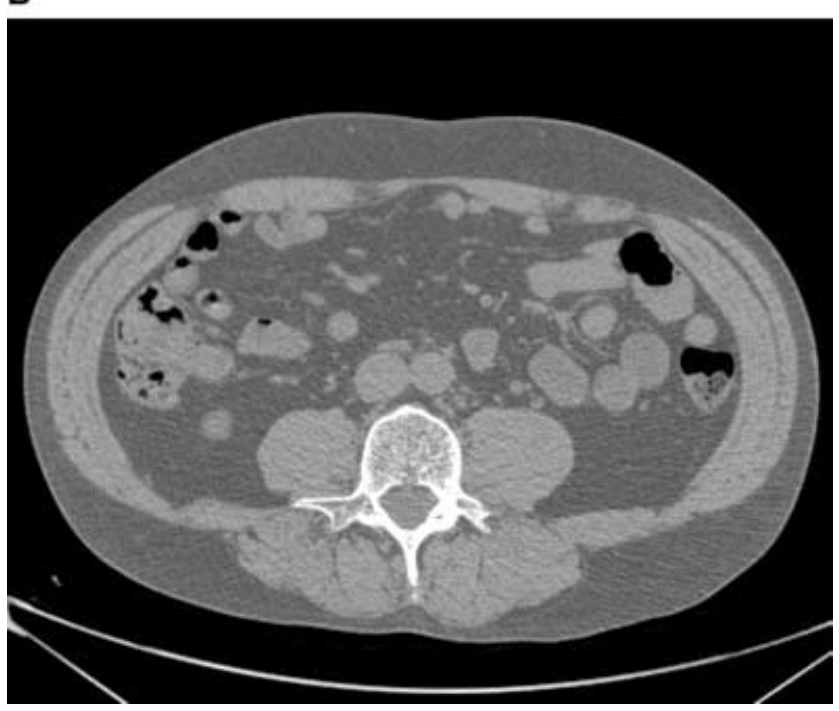

Figure 5. CT scans of patient 13 with histologic response. (A) Baseline CT-visceral fat $278.61 \mathrm{cc}$. (B) Month $12 \mathrm{CT}$ - visceral fat 166.8 cc.

flammatory grade, prompting the authors to conclude that normalization of serum ALT correlated with only mild improvement of necroinflammation (21). The other two pilot studies utilized improvement in liver histology as the primary endpoint. In one study, 18 non-diabetic patients were treated with pioglitazone for $48 \mathrm{wk}, 17$ patients completed the study. Using a strict definition of histologic response as reduction in NASH activity index (total score 12) by 3 points or greater with improvement of at least 1 point each in steatosis, parenchymal inflammation, and hepatocellular injury, 12 patients $(67 \%)$ had a histologic response. Additionally 
11 patients $(61 \%)$ had decrease in fibrosis. However, most patients gained weight, the average increase being $3.5 \mathrm{~kg}$ (15). In the other study, 22 patients were treated with $48 \mathrm{wk}$ of rosiglitazone. Significant histologic improvement was seen in the global grade, steatosis, necroinflammation, ballooning, and perisinusoidal fibrosis. Improvement in global score was observed in $13(50 \%)$ patients. An average weight gain of 6.4 $\mathrm{kg}$ was observed over the duration of treatment. Improvement in liver enzymes (AST, ALT, alkaline phosphatase, and GGT) was not sustained after treatment was discontinued (14).

Despite variations in criteria used to define histologic improvement, the proportion of patients in our study who successfully completed therapy and showed a histologic response was remarkably similar to the two recent studies on thiazolidinediones, $60 \%$ versus $67 \%$ and $59 \%(14,15)$. All three studies showed an improvement in aminotransferases and IR as well as increase in fasting HDL and decrease in serum triglycerides. Weight gain, however, is a known side effect of thiazolidinediones and the long-term effects in patients with NASH are currently unknown. Our patients had a mean weight reduction of $2.9 \mathrm{~kg}$, while patients in the two studies on thiazolidinediones had a mean weight gain of nearly $5 \mathrm{~kg}$.

The exact mechanism(s) by which dietary intervention results in histologic improvement in patients with NASH is unclear. We found that patients with histologic response had significantly greater decrease in weight and waist circumference compared to those with stable total NASH scores suggesting that weight loss and associated improvement in IR play a role in decreasing hepatic steatosis, hepatocyte ballooning, and inflammation in patients with NASH. There was also a marked reduction in visceral fat and IR in patients with histologic response compared to those with stable total NASH scores although the difference did not reach statistical significance. This was likely secondary to the small sample size of this pilot study, and a larger randomized control trial would be the logical next step to explore these results.

Not all of the patients achieved a target weight loss of 5\% total body weight, which has been recommended to achieve reduced cardiovascular risk in obesity. With our dietary intervention, food records were reviewed and counseled on how to modify their diets to achieve the macronutrient goals of the protocol. However, behavior therapy and structured exercise protocol were not used. It is possible that a higher percent of patients will achieve the target weight loss if a more structured approach for lifestyle intervention was used.

The use of lifestyle modification as an effective treatment of chronic disease is best exemplified by the Diabetes Prevention Program. This study compared strict lifestyle modification (diet and exercise) to metformin and placebo in the prevention of diabetes. Both lifestyle modification and metformin resulted in a lower incidence of diabetes compared to placebo in susceptible persons, but lifestyle modification produced a greater reduction (58\% reduction vs $31 \%$ reduction) (22). In a study of the relation of weight loss- induced change in regional adiposity to improvement in insulin sensitivity in 32 obese patients, weight loss was associated with reductions in serum triglycerides, cholesterol, and insulin levels (23). Improvement in insulin sensitivity has been shown to correlate with reduction in total body fat, especially in visceral adiposity. These data suggest that reduction in visceral adiposity is an important step in improving IR. A recent review of the effect of exercise-induced weight loss on regional distribution of body fat showed that in response to well-controlled, short-term trials, increasing physical activity expressed as energy expended is positively related to reductions in total adiposity and also reduction in abdominal and visceral fat $(24,25)$. These data corroborate our findings of a higher rate of histological response seen in patients with reductions in weight, visceral adiposity, and IR, and support the importance of visceral adiposity and IR in fatty liver disease in overweight and obese patients.

In summary, we demonstrated that 1-yr intense dietary counseling can be effective in improving liver histology in patients with biopsy-proven NASH, with results comparable to that of medications aimed to improve insulin sensitivity. We acknowledge that our study involved a very small number of highly motivated patients, so our results may not be generalized to all patients with NASH. Additionally, implementation of our program required significant personnel resources, which may not be available in routine clinical practice. A less intense but more structured approach integrating dietary counseling and exercise recommendations may be more efficacious and more easily translatable to general practice as a possible treatment for NAFLD. Finally, the improvement observed in this study may not be sustained after the program is completed as a lack of sustained benefit was observed in the pilot study utilizing rosiglitazone. Despite these limitations, we feel it is important to recommend that patients utilize dietary intervention and exercise as the first line of management in the treatment of NASH, especially if they are overweight or obese. Our findings and the recent studies on thiazolidinediones should stimulate larger scale randomized controlled trials to confirm these encouraging results and to explore practical interventions that can produce durable benefits for patients with this chronic and potentially progressive illness.

\section{ACKNOWLEDGMENT}

We would like to thank the University of Michigan GCRC for their support in funding this study (GCRC MO1-RR00042).

Reprint requests and correspondence: Hari Conjeevaram, M.D., M.S., Assistant Professor of Medicine, Division of Gastroenterology, Department of Internal Medicine, University of Michigan, 3912 Taubman Center, 1500 East Medical Center Drive, Ann Arbor, MI 48109-0362.

Received August 11, 2004; accepted December 6, 2004. 


\section{REFERENCES}

1. Kumar KS, Malet PF. Nonalcoholic steatohepatitis. Mayo Clin Proc 2000;75(7):733-9.

2. Sheth SG, Gordon FD, Chopra S. Nonalcoholic steatohepatitis. Ann Intern Med 1997;126(2):137-45.

3. Ludwig J, Viggiano TR, McGill DB, et al. Nonalcoholic steatohepatitis: Mayo Clinic experiences with a hitherto unnamed disease. Mayo Clin Proc 1980;55(7):434-8.

4. Marrero JA, Fontana RJ, Su GL, et al. NAFLD may be a common underlying liver disease in patients with hepatocellular carcinoma in the United States. Hepatology 2002;36(6):1349-54.

5. Matteoni CA, Younossi ZM, Gramlich T, et al. Nonalcoholic fatty liver disease: A spectrum of clinical and pathological severity. Gastroenterology 1999;116(6):1413-9.

6. Powell EE, Cooksley WG, Hanson R, et al. The natural history of nonalcoholic steatohepatitis: A follow-up study of forty-two patients for up to 21 years. Hepatology 1990;11(1):74-80.

7. Chitturi S, Abeygunasekera S, Farrell GC, et al. NASH and insulin resistance: Insulin hypersecretion and specific association with the insulin resistance syndrome. Hepatology 2002;35(2):373-9.

8. Pagano G, Pacini G, Musso G, et al. Nonalcoholic steatohepatitis, insulin resistance, and metabolic syndrome: Further evidence for an etiologic association. Hepatology 2002;35(2):367-72.

9. Luyckx FH, Desaive C, Thiry A, et al. Liver abnormalities in severely obese subjects: Effect of drastic weight loss after gastroplasty. Int $\mathrm{J}$ Obes Relat Metab Disord 1998;22(3):222-6.

10. Wang RT, Koretz RL, Yee HF, Jr. Is weight reduction an effective therapy for nonalcoholic fatty liver? A systematic review. Am J Med 2003;115(7):554-9.

11. Ranlov I, Hardt F. Regression of liver steatosis following gastroplasty or gastric bypass for morbid obesity. Digestion 1990;47(4):208-14.

12. Andersen T, Gluud C, Franzmann MB, et al. Hepatic effects of dietary weight loss in morbidly obese subjects. J Hepatol 1991;12(2):224-9.

13. Ueno T, Sugawara H, Sujaku K, et al. Therapeutic effects of restricted diet and exercise in obese patients with fatty liver. J Hepatol 1997;27(1):103-7.
14. Neuschwander-Tetri BA, Brunt EM, Wehmeier KR, et al. Improved nonalcoholic steatohepatitis after 48 weeks of treatment with the PPAR-gamma ligand rosiglitazone. Hepatology 2003;38(4):1008-17.

15. Promrat K, Lutchman G, Uwaifo GI, et al. A pilot study of pioglitazone treatment for nonalcoholic steatohepatitis. Hepatology 2004;39(1):188-96.

16. Reaven GM. Do high carbohydrate diets prevent the development or attenuate the manifestations (or both) of syndrome X? A viewpoint strongly against. Curr Opin Lipidol 1997;8(1):23-7.

17. Pouliot MC, Despres JP, Lemieux S, et al. Waist circumference and abdominal sagittal diameter: Best simple anthropometric indexes of abdominal visceral adipose tissue accumulation and related cardiovascular risk in men and women. Am J Cardiol 1994;73(7):460-8.

18. Katznelson L, Rosenthal DI, Rosol MS, et al. Using quantitative $\mathrm{CT}$ to assess adipose distribution in adult men with acquired hypogonadism. Am J Roentgenol 1998;170(2):4237.

19. Kugelmas M, Hill DB, Vivian B, et al. Cytokines and NASH: A pilot study of the effects of lifestyle modification and vitamin E. Hepatology 2003;38(2):413-9.

20. Hickman IJ, et al. Modest weight loss and physical activity in overweight patients with chronic liver disease results in sustained improvements in alanine aminotransferase, fasting insulin, and quality of life. Gut 2004;53(3):413-9.

21. Caldwell SH, Hespenheide EE, Redick JA, et al. A pilot study of a thiazolidinedione, troglitazone, in nonalcoholic steatohepatitis. Am J Gastroenterol 2001;96(2):519-25.

22. Knowler WC, Barrett-Connor E, Fowler SE, et al. Reduction in the incidence of type 2 diabetes with lifestyle intervention or metformin. N Engl J Med 2002;346(6):393403.

23. Goodpaster BH, Kelley DE, Wing RR, et al. Effects of weight loss on regional fat distribution and insulin sensitivity in obesity. Diabetes 1999;48(4):839-47.

24. Ross R, Dagnone D, Jones PJ, et al. Reduction in obesity and related comorbid conditions after diet-induced weight loss or exercise-induced weight loss in men. A randomized, controlled trial. Ann Intern Med 2000;133(2):92-103.

25. Ross R, Janssen I. Physical activity, total and regional obesity: Dose-response considerations. Med Sci Sports Exerc 2001;33(6 Suppl):S521-7 (Discussion S528-9). 